\title{
Multimodality and Molecular Imaging of Matrix Metalloproteinase Activation in Calcific Aortic Valve Disease
}

\author{
Jae-Joon Jung*1,2, Mahmoud Razavian*1,2, Azariyas A. Challa ${ }^{1,2}$, Lei Nie ${ }^{1,2}$, Reza Golestani ${ }^{1,2}$, Jiasheng Zhang ${ }^{1,2}$, \\ Yunpeng Ye ${ }^{1,2}$, Kerry S. Russell ${ }^{1}$, Simon P. Robinson ${ }^{3}$, Donald D. Heistad ${ }^{4}$, and Mehran M. Sadeghi ${ }^{1,2}$ \\ ${ }^{I}$ Section of Cardiovascular Medicine and Cardiovascular Research Center, Yale University School of Medicine, New Haven, \\ Connecticut; ${ }^{2}$ VA Connecticut Healthcare Systems, West Haven, Connecticut; ${ }^{3}$ Lantheus Medical Imaging, North Billerica, \\ Massachusetts; and ${ }^{4}$ Division of Cardiovascular Medicine, University of Iowa Carver College of Medicine, Iowa City, Iowa
}

Calcific aortic valve disease (CAVD) is the most common cause of aortic stenosis. Matrix metalloproteinases (MMPs) are upregulated in CAVD and contribute to valvular remodeling and calcification. We investigated the feasibility and correlates of MMP-targeted molecular imaging for detection of valvular biology in CAVD. Methods: Apolipoprotein E-deficient $\left(\mathrm{apoE}^{-/-}\right)$mice were fed a Western diet (WD) for 3, 6, and $9 \mathrm{mo}(n=108)$ to induce CAVD. Wild-type mice served as the control group $(n=24)$. The development of CAVD was tracked with CT, echocardiography, MMP-targeted smallanimal SPECT imaging using ${ }^{99 m T c-R P 805, ~ a n d ~ h i s t o l o g i c ~ a n a l y s i s . ~}$ Results: Key features of CAVD-leaflet thickening and valvular calcification-were noted after 6 mo of WD and were more pronounced after 9 mo. These findings were associated with a significant reduction in aortic valve leaflet separation and a significant increase in transaortic valve flow velocity. On in vivo SPECT/CT images, MMP signal in the aortic valve area was significantly higher at $6 \mathrm{mo}$ in WD mice than in control mice and decreased thereafter. The specificity of the signal was demonstrated by blocking, using an excess of nonlabeled precursor. Similar to MMP signal, MMP activity as determined by in situ zymography and valvular inflammation by CD68 staining were maximal at 6 mo. In vivo ${ }^{99 m T c-R P 805 ~ u p-~}$ take correlated significantly with MMP activity $\left(R^{2}=0.94, P<0.05\right)$ and CD68 expression $\left(R^{2}=0.98, P<0.01\right)$ in CAVD. Conclusion: MMP-targeted imaging detected valvular inflammation and remodeling in a murine model of CAVD. If this ability is confirmed in humans, the technique may provide a tool for tracking the effect of emerging medical therapeutic interventions and for predicting outcome in CAVD.

Key Words: molecular imaging; aortic valve; inflammation; calcification; matrix metalloproteinases

J Nucl Med 2015; 56:933-938

DOI: 10.2967/jnumed.114.152355

\section{A}

ortic stenosis is the most common cause of valvular heart disease, and calcific aortic valve disease (CAVD) is the most common cause of aortic stenosis (1). Currently, treatment for CAVD is

\footnotetext{
Received Dec. 7, 2014; revision accepted Apr. 6, 2015.

For correspondence or reprints contact: Mehran M. Sadeghi, VA Connecticut Healthcare System, 950 Campbell Ave., 111B, West Haven, CT 06516.

E-mail: mehran.sadeghi@yale.edu

${ }^{*}$ Contributed equally to this work.

Published online Apr. 23, 2015.

COPYRIGHT (c) 2015 by the Society of Nuclear Medicine and Molecular Imaging, Inc.
}

limited to valve replacement, valvuloplasty, or transcatheter aortic valve replacement for symptomatic stenosis. Several medical treatments evaluated for slowing or reversing the pathogenic process in CAVD have proven ineffective in clinical trials $(2-4)$. It is speculated that this lack of efficacy may be due to initiation of therapeutic interventions at an irreversible stage of CAVD or to the difficulty of demonstrating treatment efficacy when there are a large number of low-risk patients and only a fraction of the patients with aortic sclerosis develop symptomatic aortic valve stenosis. The high prevalence of aortic sclerosis in the general population $(\sim 25 \%$ in those older than 65 y (5)) suggests that early diagnosis, by itself, may not be sufficient and that it is important to identify those patients who would most benefit from preventive and therapeutic interventions. Molecular imaging targeted at relevant aspects of aortic valve biology can lead to detection of processes that determine disease progression, ultimately facilitating drug development and early assessment of therapeutic interventions.

Several aspects of aortic valve biology have been targeted in murine and rabbit models of CAVD by ex vivo near-infrared fluorescent imaging and MR imaging and spectroscopy (6-8). Although useful for mechanistic studies on animal models, these approaches have a limited, if any, possibility of translation to clinical imaging in humans using current imaging technology. Reports on ${ }^{18} \mathrm{~F}-\mathrm{FDG}$ and ${ }^{18} \mathrm{~F}-\mathrm{NaF}$ PET imaging of the aortic valve demonstrated the feasibility of noninvasive imaging of valvular biology by nuclear imaging in humans $(9,10)$. However, ${ }^{18} \mathrm{~F}-\mathrm{FDG}$ imaging is notoriously nonspecific, and the clinical value of ${ }^{18} \mathrm{~F}-\mathrm{NaF}$ imaging, which detects the calcification process, remains to be fully established. Given the key role of matrix metalloproteinases (MMPs) in valvular remodeling and inflammation and their high levels of expression in human CAVD (11), we investigated the feasibility of MMP-targeted small-animal SPECT/CT imaging for in vivo detection of valvular biology and structure in a murine model of CAVD.

\section{MATERIALS AND METHODS}

Please refer to the supplemental data for additional details on the materials and methods (supplemental materials are available at http:// jnm.snmjournals.org).

\section{Animal Model}

Four- to 5-wk-old apolipoprotein E-deficient (apoE ${ }^{-l-}$ ) mice (originally from Jackson Laboratory) were fed a high-fat Western diet (WD, $0.15 \%$ cholesterol and $40 \%$ calories from fat) for 2-4 mo ("3-mo group," $n=25$ ), 5-7 mo ("6-mo group," $n=45$ ), and 8-10 mo ("9-mo group," $n=38$ ). Wild-type mice were fed normal chow for 3-6 mo and 
served as the control group $(n=24)$. The experimental scheme is presented in Supplemental Figure 1. All experiments were performed according to the regulations of the institutional Animal Care and Use Committees of Yale University and VA Connecticut.

\section{Imaging}

Echocardiographic images were obtained using a Vevo 2100 system (VisualSonics) with a 30-MHZ probe. RP805, a ${ }^{99 m}$ Tc-labeled tracer with specificity for activated MMPs, was provided by Lantheus Medical Imaging and labeled as previously described (12). SPECT/CT was performed and analyzed as described previously, with minor modifications, using a high-resolution small-animal imaging system (X-SPECT; Gamma Medica) (13-15). After completion of in vivo imaging, the heart (with aortic valve) and aorta were harvested for ex vivo planar imaging and $\gamma$-well counting.

\section{Statistical Analysis}

Values from continuous parameters are presented as mean \pm SE. Data from 2 groups were compared using the Student $t$ test. Multiple groups were compared using 1-way ANOVA followed by the Bonferroni multiple-comparison test. The linear relationship between 2 variables was addressed using the Pearson correlation coefficient. The level of significance was set at a $P$ value of less than 0.05 .

\section{RESULTS}

\section{CAVD in ApoE ${ }^{-/-}$Mice}

To induce CAVD, we fed apoE $\mathrm{E}^{-1-}$ mice a WD for up to 9 mo. The development of valvular calcification in these mice was monitored by combining unenhanced CT with contrast-enhanced CT to localize the aortic valve while the mouse was kept in the same position (Fig. 1A). Although no valvular calcification was detectable on in vivo CT images of control mice or 3-mo WD mice, valvular calcification could be seen in a subset (17\%) of mice after 6 mo of WD, and the incidence of detectable calcification increased to $50 \%$ after 9 mo of WD. To investigate whether these mice develop aortic stenosis, we assessed aortic valve anatomy and physiology using echocardiography. Compared with control mice, 6- and 9-mo WD mice showed significantly reduced leaflet separation $(1.17 \pm 0.03,1.02 \pm 0.04,0.99 \pm 0.04$, and $0.96 \pm$ $0.05 \mathrm{~mm}$ for control mice and 3-, 6-, and 9-mo WD mice, respectively; $n=14-23$ in each group, $P<0.05$ for both) (Fig. 1B and Supplemental Fig. 2A). When significant aortic stenosis was defined as maximum leaflet separation less than the mean minus 2.5 SDs of that in control mice, the incidence of significant aortic stenosis in WD mice increased from $15.8 \%$ and $17.4 \%$ at 3 and 6 mo, respectively, to $30 \%$ at 9 mo. The reduction in leaflet separation at 9 mo in WD mice relative to control mice was associated with a significant increase in peak flow velocity across the aortic valve (but not in the left ventricle outflow tract) (Fig. 1C and Supplemental Fig. 2B). Histologic analysis of aortic valve tissue showed a gradual thickening of leaflets over time in WD mice. As such, relative to the leaflets of control mice or 3-mo WD mice, leaflet thickness was significantly greater after 6 mo of WD and further increased after 9 mo $(n=3$ in each group, $P<0.001)$ (Fig. 2A). Consistent with CT data, alizarin red staining showed a gradual increase in valvular calcification over time in WD mice, with considerable calcification detectable after 6 mo. Indeed, the percentage of alizarin red-positive area in the aortic valves was significantly higher in 6- and 9-mo WD mice than in control mice or 3-mo WD mice $(n=3$ in each group: $P<0.01$ for 6 -mo WD vs. control or 3-mo WD, and $P<0.001$ for 9- vs. 6-mo WD) (Fig. 2B).

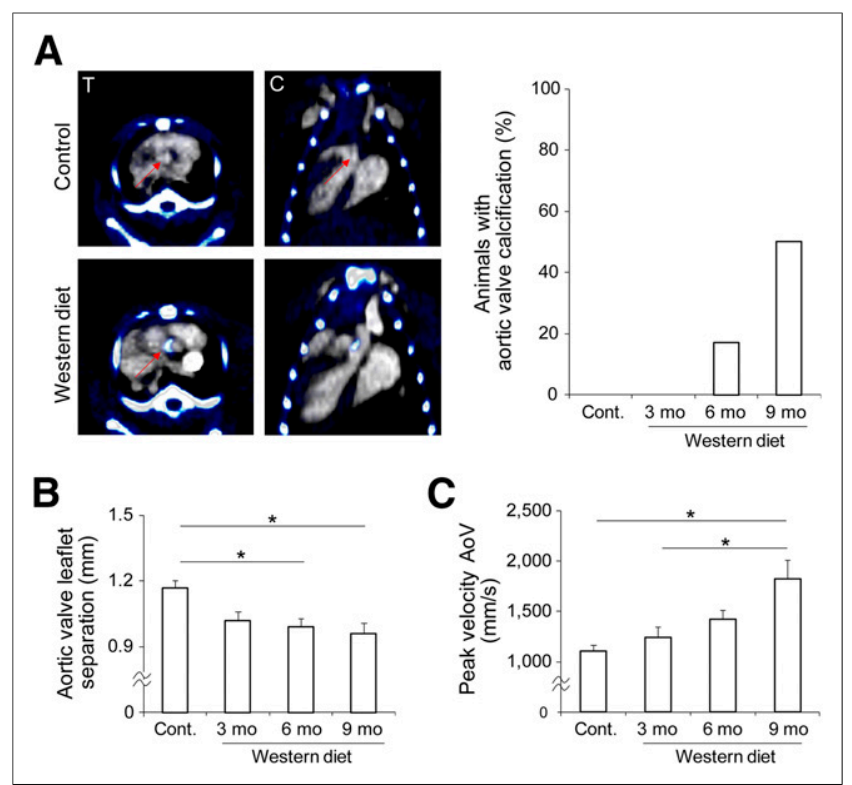

FIGURE 1. CAVD development in apoE ${ }^{-/-}$mice. (A) Examples of fused unenhanced (pseudocolored) and contrast-enhanced CT images of control mouse (top left panels) and 6-mo WD mouse (bottom left panels) demonstrating aortic valve calcification (arrows) in WD-fed mouse; percentage of mice with CT-detectable aortic valve calcification (right panel). $\mathrm{C}=$ coronal; $\mathrm{T}=$ transverse. $n=7,6,23$, and 16 for control and 3, 6, and 9 mo of WD, respectively. (B) Quantification of M-mode echocardiography-derived systolic aortic valve leaflet separation. ${ }^{\star} P<$ $0.05, n=14-23$ in each group. (C) Echo Doppler-derived peak systolic flow velocity across aortic valve (AoV). ${ }^{\star} P<0.05, n=14-23$ in each group.

\section{MMP Imaging of CAVD}

To investigate the feasibility of MMP-targeted imaging in murine CAVD, the control and WD mice underwent SPECT imaging with 99mTc-RP805, a tracer with specificity for activated MMPs. SPECT was combined with contrast-enhanced CT to identify anatomic structures. ${ }^{99 \mathrm{~m} T c-R P 805}$ signal in the aortic valve area was best detected in 6-mo WD mice (Fig. 3 and Supplemental Fig. 3). Quantification of aortic valve ${ }^{99 \mathrm{~m}} \mathrm{Tc}-\mathrm{RP} 805$ signal demonstrated significantly higher uptake in 6-mo WD mice than in control or 3-mo WD mice $(13 \pm 2,19 \pm 3,44 \pm 6$, and $31 \pm 6$ counts per voxel/kBq of injected dose for control mice and 3-, 6-, and 9-mo WD mice, respectively $[n=7-13$ in each group, $P<0.0001$ and $<0.01$ for control and 3-mo WD, respectively]). Despite their higher level of valvular calcification, 9-mo WD mice showed a nonstatistically significant trend toward less aortic valve ${ }^{99 \mathrm{~m}} \mathrm{Tc}-\mathrm{RP} 805$ uptake than 6-mo WD mice.

99mTc-RP805 uptake in the aortic valve was confirmed by ex vivo planar imaging (Fig. 4A). Consistent with the in vivo imaging results, ${ }^{99 \mathrm{~m}} \mathrm{Tc}-\mathrm{RP} 805$ uptake in the aortic valve area was significantly higher in 6-mo WD mice than in control or 3-mo WD mice $(299 \pm 25,366 \pm 43,707 \pm 90$, and $535 \pm 56$ counts per pixel/ $\mathrm{kBq}$ of injected dose for control mice and 3-, 6-, and 9-mo WD, respectively $[n=9-15$ in each group, $P<0.001$ and $<0.05$ for control and 3-mo WD, respectively]). Importantly, in vivo quantification of ${ }^{99 \mathrm{~m}} \mathrm{Tc}-\mathrm{RP} 805$ uptake correlated well with its ex vivo quantification $\left(R^{2}=0.52, P<0.0001\right.$, Fig. 4B). The small size of the valve and its leaflets did not allow more refined localization of ${ }^{99 m}$ Tc-RP805 uptake within the leaflets or base of the valve by planar imaging. Therefore, in a subset of mice we quantified tracer 


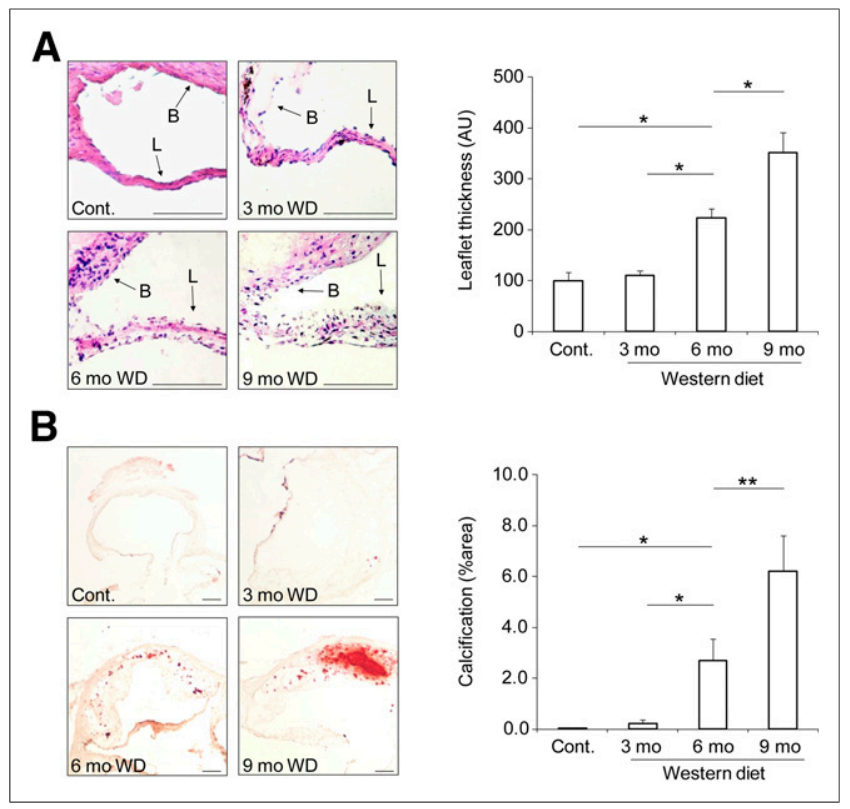

FIGURE 2. Histologic analysis of CAVD in apoE ${ }^{-/-}$mice. (A) Examples of aortic valve leaflet hematoxylin and eosin staining (left panels) and quantification of aortic valve leaflet thickness (right panel). Scale bar $=$ $100 \mu \mathrm{m} . \mathrm{AU}=$ arbitrary units; $\mathrm{B}=$ base; $\mathrm{L}=$ leaflet. ${ }^{\star} P<0.001, n=3$ in each group. (B) Examples (left panels) and quantification (right panel) of alizarin red staining of aortic valve calcification. Scale bar $=100 \mu \mathrm{m}$. ${ }^{\star} P<0.01,{ }^{\star \star} P<0.001, n=3$ in each group.

uptake in the leaflets and base by $\gamma$-well counting. A significant correlation between ${ }^{99 \mathrm{~m}} \mathrm{Tc}-\mathrm{RP} 805$ signal in the leaflets and the base ( $n=9, R^{2}=0.47, P<0.05$, data not shown) indicated tracer was taken up by the entire valve apparatus. To investigate the specificity of tracer uptake, we administered a 400-fold excess of unlabeled precursor to a group of four 6-mo WD mice immediately before ${ }^{99 \mathrm{~m}} \mathrm{Tc}-\mathrm{RP} 805$ administration. In vivo SPECT/CT and ex vivo planar imaging showed a significant reduction in ${ }^{99 \mathrm{~m} T c-R P 805}$ uptake in the aortic valve after preadministration of excess precursor, indicating the specificity of ${ }^{99 \mathrm{~m} T c-R P 805}$ uptake in this model $(P<0.05$ for both, Figs. 5A and 5B).

\section{Correlates of MMP Signal in CAVD}

In line with the role of MMP activation in valvular remodeling and stenosis, the temporal pattern of MMP signal in CAVD (which was maximal at $6 \mathrm{mo}$ ) was different from the pattern of aortic valve leaflet separation, flow velocity, and calcification. MMP-12 is the most upregulated MMP gene in CAVD (11). In the absence of reliable antimurine MMP-12 antibodies (or other antimurine MMP antibodies) for immunostaining, we relied on quantitative reverse-transcription polymerase chain reaction to assess MMP-12 expression in our model. Similar to the ${ }^{99 \mathrm{~m} T c-R P 805}$ uptake pattern, MMP-12 expression was maximal in mice after 6 mo of WD ( $n=3-5$ in each group: $P<0.0001$ for $6-$ mo WD vs. control, and $P<0.05$ for 6- vs. 3-mo WD, Fig. 6A). Furthermore, there was a significant correlation between in vivo ${ }^{99 \mathrm{~m}} \mathrm{Tc}$-RP805 uptake and MMP-12 expression $\left(R^{2}=0.93, P<0.05\right.$, Fig. 6B). Next, we assessed MMP activity in the aortic valve using in situ zymography with a fluorogenic pan-MMP substrate. Aortic valve MMP activity was maximal in 6-mo WD mice $(n=3$ in each group: $P<$ 0.01 for 6 -mo WD vs. control, and $P<0.05$ for $6-$ vs. 3 -mo WD, Fig. 6C). Aortic valve leaflet MMP activity as detected by high- resolution confocal microscopy paralleled that of the entire valve, with the highest activity being seen in the leaflets of 6-mo WD mice (Supplemental Fig. 4A). Consistent with ${ }^{99 m}$ Tc-RP805 targeting of MMP activation, there was a significant correlation between in vivo ${ }^{99 \mathrm{~m}} \mathrm{Tc}-\mathrm{RP} 805$ uptake in CAVD and MMP activity quantified by in situ zymography $\left(R^{2}=0.94, P<0.05\right.$, Supplemental Fig. 4B). Because inflammation is a major contributor to MMP production and activation, we examined the macrophage content of the aortic valve in the course of CAVD development. In the aortic valves of control mice, few, if any, macrophages could be detected by CD68 immunostaining (Fig. 7A). In 3-mo WD mice, CD68 staining was detectable at low levels. Following a temporal pattern similar to that of ${ }^{99 \mathrm{~m}} \mathrm{Tc}-\mathrm{RP} 805$ uptake, aortic valve CD68 expression was maximal at 6 mo of WD and decreased by 9 mo with progression of valvular calcification $(n=$ 3 in each group: $P<0.001$ for 6 -mo WD vs. control, and $P<$ 0.01 for 6- vs. 3-mo WD). A significant correlation was found between in vivo ${ }^{99 \mathrm{~m}} \mathrm{Tc}-\mathrm{RP} 805$ uptake and CD68 expression $\left(R^{2}=\right.$ $0.98, P<0.01$, Fig. 7B).

\section{DISCUSSION}

Our data demonstrate the development of CAVD in apoE $\mathrm{E}^{-1-}$ mice fed a WD for 9 mo, establish the feasibility of MMP-targeted small-animal SPECT/CT imaging of CAVD, and define the correlates of MMP activation in the aortic valve, thus demonstrating a

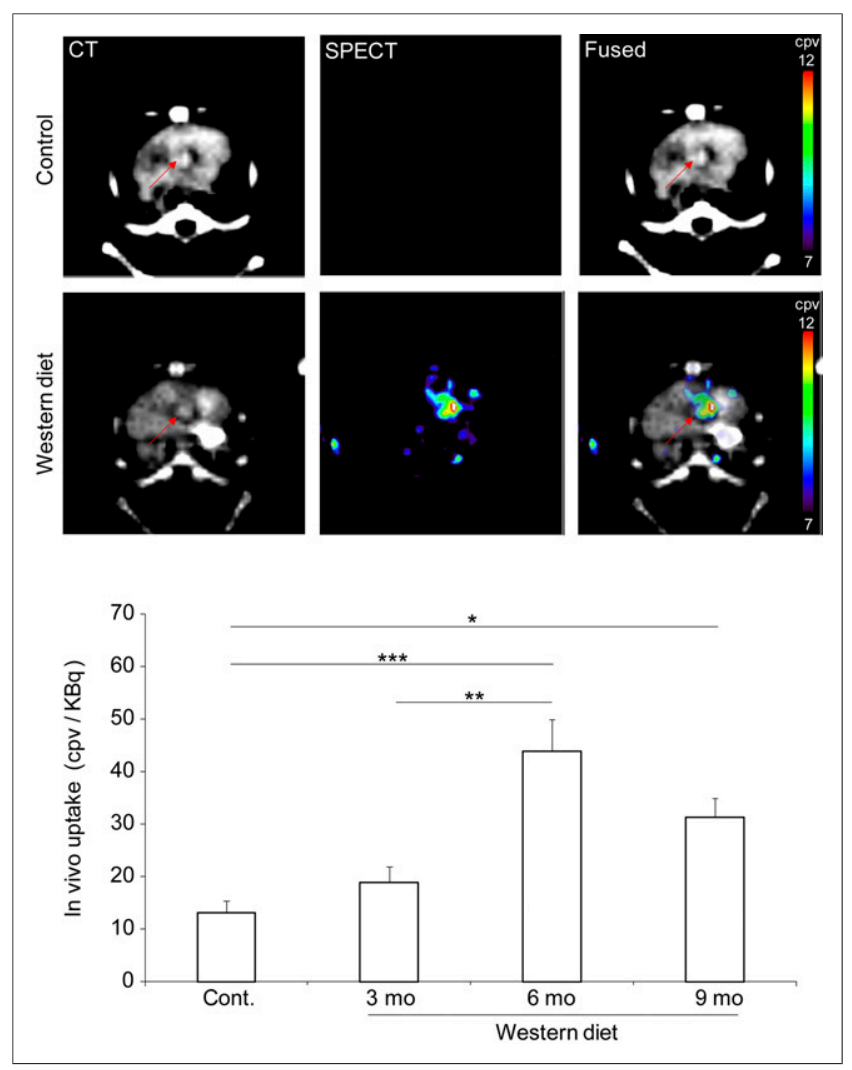

FIGURE 3. In vivo imaging of MMP activation in CAVD. Examples of in vivo CT angiography, 99mTc-RP805 SPECT, and fused transverse images in control mouse and 6-mo WD mouse (top panels); backgroundcorrected quantification of ${ }^{99 \mathrm{~m} T c-R P 805}$ uptake in CAVD (bottom panel). Arrows point to aortic valve area. $\mathrm{cpv}=$ counts per voxel. ${ }^{\star} P<0.05$, ${ }^{\star *} P<0.01,{ }^{\star \star \star} P<0.0001, n=7-13$ in each group. 


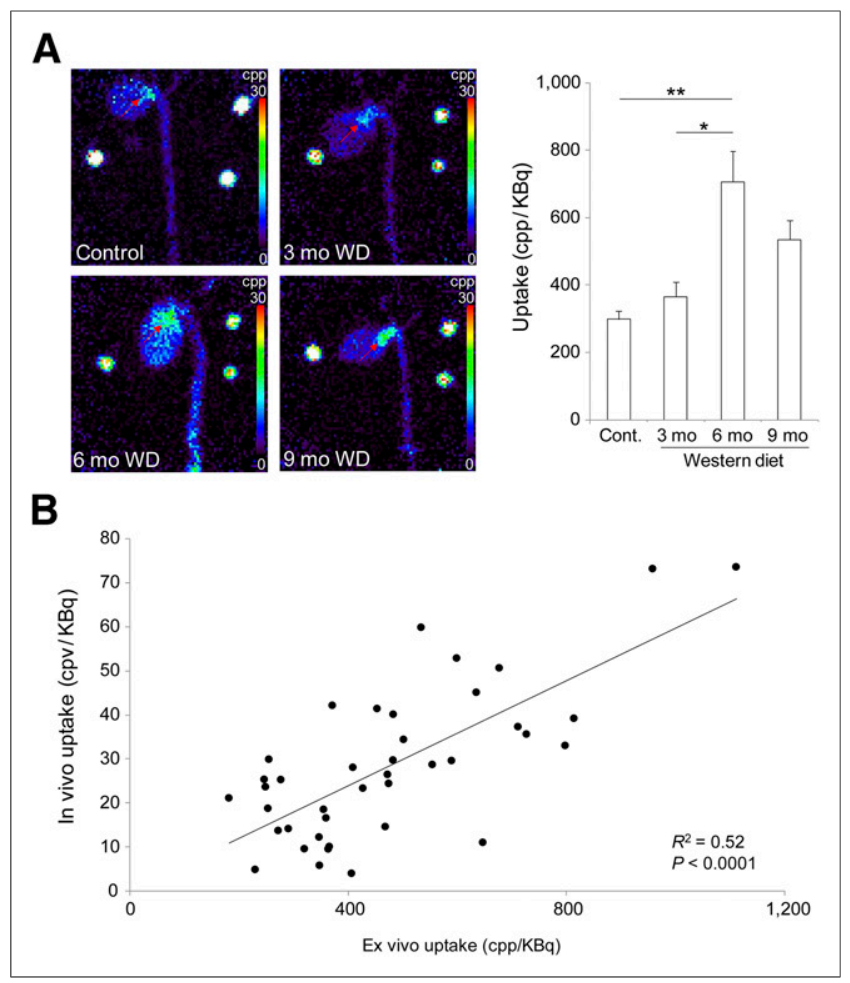

FIGURE 4. EX vivo imaging of MMP activation in CAVD. (A) Examples of ex vivo $99 \mathrm{mTc}-\mathrm{RP} 805$ planar images of heart and aorta in control mouse and WD mouse (left panels); background-corrected quantification of $99 \mathrm{mTC}-\mathrm{RP} 805$ uptake in CAVD (right panel). Arrows point to aortic valve area. Three point sources are seen in field of view. ${ }^{*} P<0.01$, ${ }^{* \star} P<0.001, n=9-15$ in each group. (B) Correlation between in vivo and ex vivo quantification of ${ }^{99 m T c-R P 805}$ uptake in CAVD. $c p p=$ counts per pixel; $\mathrm{cpv}=$ counts per voxel.

potential application of MMP-targeted imaging for tracking valvular remodeling in CAVD. Traditionally, the focus of clinicians who evaluate CAVD patients has been on detection and quantification of valvular stenosis. Somewhat similar to the relation between atherosclerosis and plaque rupture, high-grade stenosis is the outcome in only a small subset of patients with CAVD. In addition, many patients with severe CAVD do not have critical aortic valve stenosis whereas others with less advanced disease become symptomatic. As such, CAVD and symptomatic valvular stenosis are distinct yet related concepts. In vivo detection and quantification of the pathogenic processes of CAVD is a prerequisite to develop and evaluate novel therapies that prevent progression of CAVD.

Murine models of CAVD are based on genetic or dietary interventions and lead to varying degrees of aortic stenosis (16). Here, we have shown in WD-fed apoE ${ }^{-1-}$ mice that the aortic valve leaflets thicken over time and that nearly half the mice develop CT-detectable valvular calcification by 9 mo. This valvular remodeling is associated with a significant reduction in leaflet separation and a significant increase in aortic valve peak flow velocity. Accurate evaluation of aortic stenosis in humans can be difficult, and evaluation of aortic stenosis in mice is also a rather challenging task, with many pitfalls. The main echocardiographybased methods to evaluate aortic valve anatomy and function in mice are M-mode echocardiography, which provides the necessary spatial and temporal resolution to assess aortic valve leaflet separation and has been validated against invasive measurement of the transvalvular pressure gradient (16), and Doppler to estimate the transvalvular pressure gradient and valve area. Doppler measurements alone can be influenced by confounders such as aortic regurgitation, left ventricular function, and cardiac output. However, the combination of changes in M-mode measurement of aortic valve leaflet separation and transvalvular Doppler velocity in the absence of a significant change in left ventricular outflow track velocity indicates the development of aortic stenosis in WD-fed apoE ${ }^{-1-}$ mice.

Inflammation, extracellular matrix remodeling (including fibrosis), and calcification are hallmarks of CAVD $(17,18)$. Normal aortic valve leaflets are covered by an endothelial layer and contain several extracellular matrix proteins (e.g., collagen, elastin, fibronectin, and laminin), proteoglycans, and valvular interstitial cells. There are few resident leukocytes (mainly macrophages) in the normal aortic valve (17). Mechanical stress and deposition of lipid particles, among other factors, induce focal inflammation, which is believed to play a central role in the pathogenesis of CAVD (19). As such, inflammation triggers osteoblastic transformation of valvular interstitial cells, and ultimately valvular calcification. Proinflammatory cytokines produced by inflammatory cells trigger valvular MMP expression and matrix remodeling (18).

MMPs are a multigene family of endopeptidases that selectively digest individual components of the extracellular matrix (20). Most secreted MMPs are released into the extracellular space in a latent or proenzyme state. Activation of these latent MMPs exposes the catalytic domain targeted by ${ }^{99 \mathrm{~m} T c-R P 805}$, the tracer used in our studies. Recent data show that activated MMPs bind to, and can be taken up by, neighboring cells (21). Several MMPs are expressed in CAVD and may contribute to valvular fibrosis and calcification (22-24). Indeed, expression profiling of healthy and

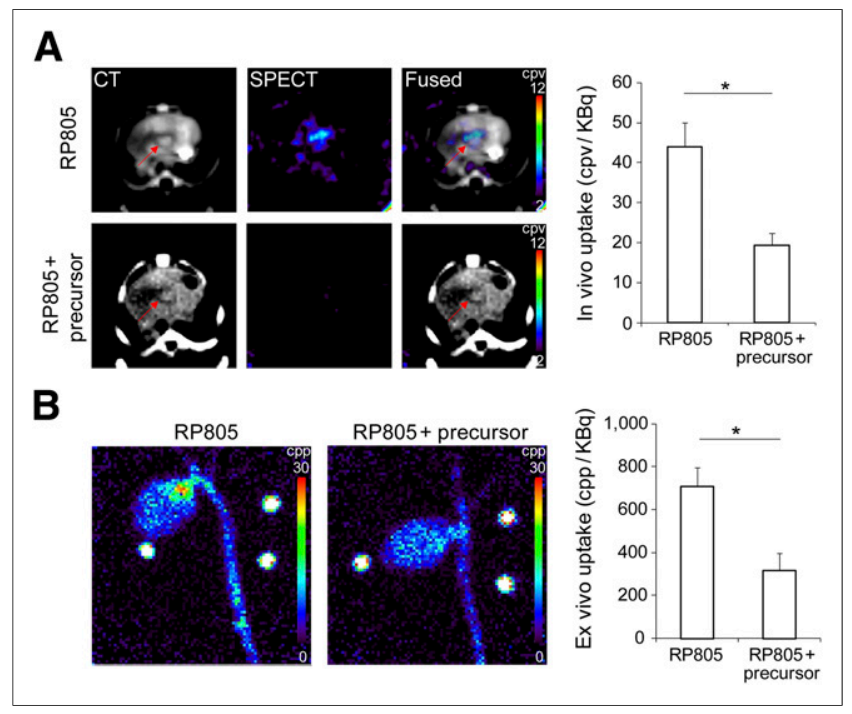

FIGURE 5. Specificity of ${ }^{99 m}$ Tc-RP805 uptake in CAVD. (A) Examples of in vivo CT angiography, ${ }^{99 m T c-R P} 805$ SPECT, and fused transverse images in mice fed WD for 6 mo without (top row) and with (bottom row) preadministration of excess unlabeled precursor (left panels); background-corrected quantification of 99mTc-RP805 uptake in CAVD without $(n=10)$ and with $(n=4)$ preadministration of excess nonlabeled precursor (right panel). Arrows point to aortic valve area. $\mathrm{cpv}=$ counts per voxel. ${ }^{\star} P<0.05$. (B) Examples (left panels) and quantification (right panel) of ${ }^{99 m T c-R P 805}$ uptake on ex vivo planar images in mice fed WD for 6 mo without $(n=13)$ or with $(n=4)$ preadministration of excess nonlabeled precursors. $\mathrm{cpp}=$ counts per pixel. ${ }^{*} P<0.05$. 


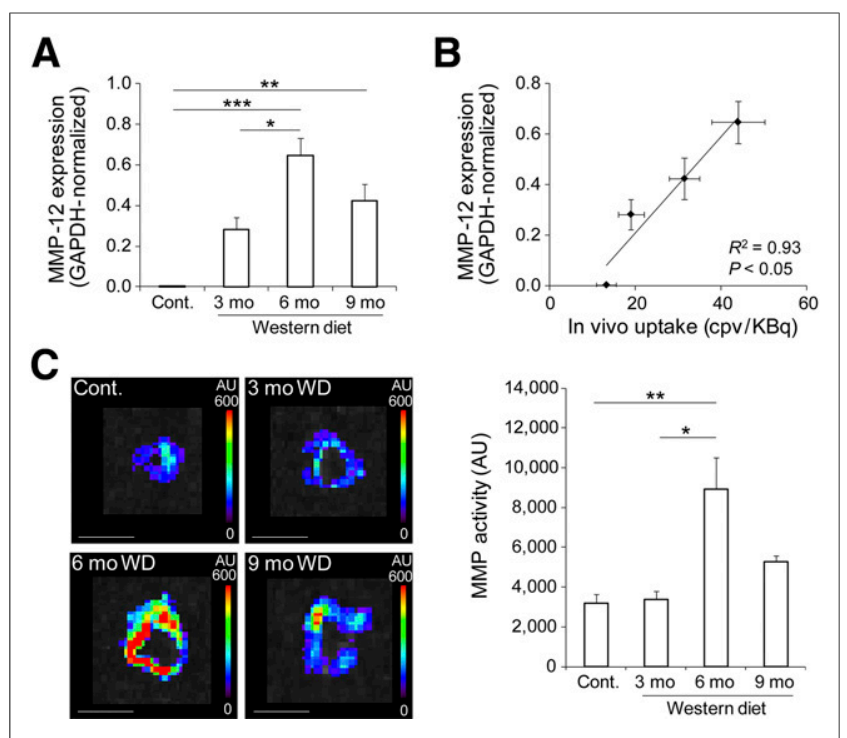

FIGURE 6. MMP expression and activation in CAVD. (A) GAPDHnormalized MMP-12 expression assessed by quantitative reversetranscription polymerase chain reaction in CAVD. ${ }^{\star} P<0.05$, ${ }^{\star \star} P<0.01,{ }^{\star \star *} P<0.0001, n=3-5$ in each group. (B) Correlation between MMP-12 expression and in vivo ${ }^{99 m T c-R P 805}$ uptake in CAVD. $\mathrm{cpv}=$ counts per voxel. $(\mathrm{C})$ Representative examples (left panels) and quantification (right panel) of MMP activation detected by in situ zymography in CAVD. Scale bar $=4 \mathrm{~mm}$. AU = arbitrary units. ${ }^{*} P<0.05,{ }^{* *} P<0.01, n=3$ in each group.

(rather advanced) calcific human aortic valves has identified several MMPs as among the top 10 upregulated genes in CAVD and MMP-12 as the most upregulated (11). This finding led us to investigate the temporal pattern of MMP-12 expression in our model. MMP-12 expression peaked at 6 mo of WD, before the development of more advanced calcific lesions, raising the possibility of a similar finding in humans. Potential sources of MMP production and activation in CAVD are inflammatory cells, valvular interstitial cells, and endothelial cells (17). Several mediators of valvular inflammation and remodeling, including cytokines, periostin, oxidative stress, and hemodynamics, regulate MMP expression and activation $(25,26)$. Similarly, several key regulators of aortic valve calcification, including transforming growth factor $\beta 1$ and receptor activator of nuclear factor $\kappa-B$ ligand, as well as mechanical stretch, can induce MMP activation in cell or organ cultures (27-29). As such, MMP expression and activation appear to be closely linked to valvular remodeling and calcification in CAVD, highlighting the potential of MMP-targeted imaging for detection of the remodeling process in CAVD.

Some aspects of CAVD biology have been targeted by ex vivo optical imaging in mice, and these studies have provided important insight into aortic valve pathobiology $(6,7)$. However, noninvasive optical imaging of CAVD lacks a clear path to clinical translation. Unlike optical imaging, nuclear imaging techniques are highly quantitative and can readily be applied to noninvasive imaging in humans. As a first step toward potential clinical applications, in this in vivo imaging study we demonstrated the feasibility of SPECT/CT-based MMP-targeted imaging of CAVD in mice. CAVD is often associated with atherosclerosis, and there is ongoing debate on their similarities and differences. The differentiation of leaflet-specific processes from those at the base is impossible and without any practical implication. We found a good correlation between tracer uptake in the leaflets and tracer uptake in the base of the valve, and MMP activity in the leaflets paralleled MMP tracer uptake. Together, these data indicate that various components of the valve apparatus contribute to valvular ${ }^{99 m} \mathrm{Tc}-\mathrm{RP} 805$ signal in vivo. We ascertained the validity of our in vivo quantification methodology by correlating the data obtained in vivo with the results of ex vivo imaging. The time course of MMP activation in CAVD suggests that MMP-targeted imaging can be used for tracking the remodeling process in vivo. Indeed, peak MMP activation in the aortic valve was noted at 6 mo, preceding the development of advanced valvular calcification and stenosis. Not surprisingly, MMP activation in the valve paralleled CD68 expression, suggesting that it may also be useful as a surrogate marker for early assessment of the effect of emerging therapeutic interventions aimed at preventing progression of the disease.

The failure of lipid-lowering, antiinflammatory treatments in preventing the progression of CAVD in patients with mild to moderate stenosis (2-4) may be due to limited understanding of the pathophysiology or to poor selection of patients who might benefit from such interventions. Molecular imaging provides an investigational tool for mechanistic studies of aortic valve disease. Unique to the noninvasive approaches studied here, nuclear-based molecular imaging allows for serial assessment of aortic valve biology in the same patient. Our data suggest that MMP-targeted molecular imaging may be used to detect valvular inflammation, potentially identifying those patients prone to more rapid disease progression. The ability to do so would have practical implications regarding the choice of therapeutic intervention, such as when a patient with moderate aortic stenosis is to undergo bypass surgery. In addition, this ability could help focus clinical trials on those patients who would most benefit from emerging therapies, as well as helping to track the effectiveness of interventions. Given

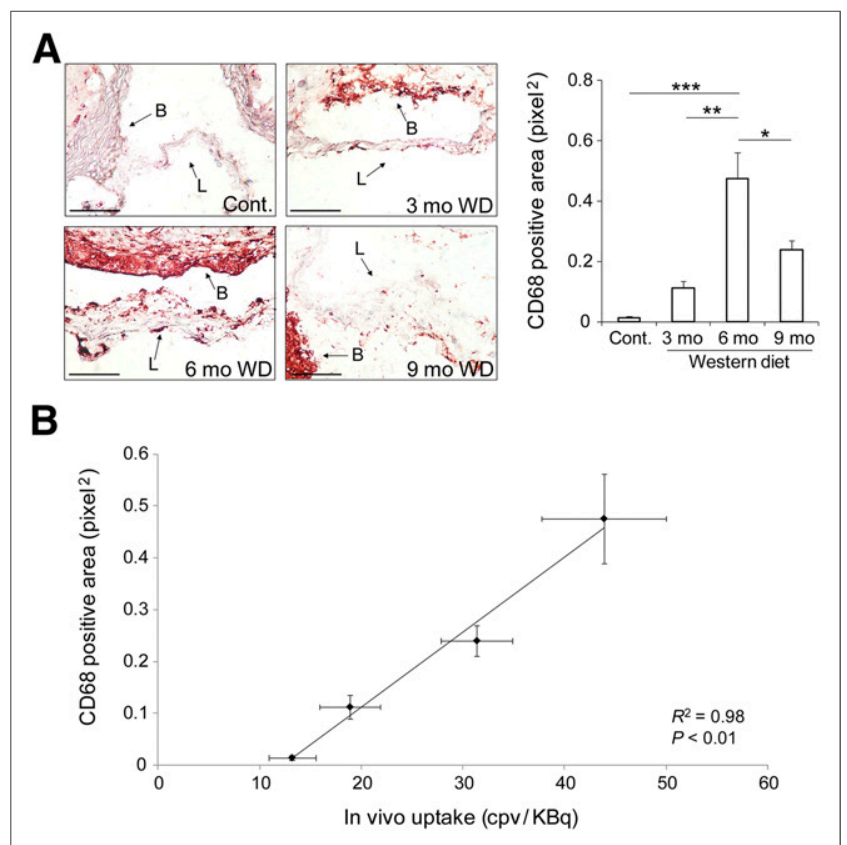

FIGURE 7. Inflammation in CAVD. (A) Examples (left panels) and quantification (right panel) of CD68 expression detected by immunohistochemistry in CAVD. Scale bar $=100 \mu \mathrm{m}$. B = base; $\mathrm{L}=$ leaflet. ${ }^{*} P<$ $0.05,{ }^{\star \star} P<0.01,{ }^{\star \star \star} P<0.001, n=3$ in each group. (B) Correlation between CD68 expression and in vivo ${ }^{99 m T C-R P 805}$ uptake in CAVD. 
the absence of optimal large-animal models of CAVD, it is likely that clinical implementation of this approach requires direct validation in patients with early-stage aortic stenosis. The cost and added value are among factors to consider in bringing a new imaging technique to the clinic. Approaches such as MMP imaging that target fundamental biologic processes (e.g., remodeling and inflammation) have a broad range of potential clinical applications, and their further development for clinical use is therefore more economically viable.

\section{CONCLUSION}

The multimodality imaging approach to CAVD anatomy, physiology, and biology used here provides a comprehensive picture of CAVD. If validated in humans, this approach could have a major impact on the management of patients with CAVD.

\section{DISCLOSURE}

The costs of publication of this article were defrayed in part by the payment of page charges. Therefore, and solely to indicate this fact, this article is hereby marked "advertisement" in accordance with 18 USC section 1734 . This work was supported by National Institutes of Health R01 HL112992 and R01 HL114703 and by Department of Veterans Affairs Merit Awards I0-BX001750 and P01 HL062984. Simon Robinson is an employee of Lantheus Medical Imaging. Mehran M. Sadeghi received experimental tracers from Lantheus Medical Imaging. No other potential conflict of interest relevant to this article was reported.

\section{ACKNOWLEDGMENT}

We thank Nicole Mikush for performing and assisting in reading the echocardiograms.

\section{REFERENCES}

1. Roger VL, Go AS, Lloyd-Jones DM, et al. Heart disease and stroke statistics: 2011 update-a report from the American Heart Association. Circulation. 2011;123:e18-e209.

2. Cowell SJ, Newby DE, Prescott RJ, et al. A randomized trial of intensive lipidlowering therapy in calcific aortic stenosis. N Engl J Med. 2005;352:23892397.

3. Rosseb $\varnothing \mathrm{AB}$, Pedersen TR, Boman K, et al. Intensive lipid lowering with simvastatin and ezetimibe in aortic stenosis. N Engl J Med. 2008;359: 1343-1356.

4. Chan KL, Teo K, Dumesnil JG, Ni A, Tam J. Effect of lipid lowering with rosuvastatin on progression of aortic stenosis: results of the Aortic Stenosis Progression Observation: Measuring Effects of Rosuvastatin (ASTRONOMER) Trial. Circulation. 2010;121:306-314

5. Stewart BF, Siscovick D, Lind BK, et al. Clinical factors associated with calcific aortic valve disease: cardiovascular health study. J Am Coll Cardiol. 1997;29:630-634.

6. Aikawa E, Nahrendorf M, Sosnovik D, et al. Multimodality molecular imaging identifies proteolytic and osteogenic activities in early aortic valve disease. Circulation. 2007;115:377-386.
7. Aikawa E, Aikawa M, Libby P, et al. Arterial and aortic valve calcification abolished by elastolytic cathepsin S deficiency in chronic renal disease. Circulation. 2009;119:1785-1794.

8. Waters EA, Chen J, Allen JS, Zhang H, Lanza GM, Wickline SA. Detection and quantification of angiogenesis in experimental valve disease with integrin-targeted nanoparticles and 19-fluorine MRI/MRS. J Cardiovasc Magn Reson. 2008;10:43.

9. Marincheva-Savcheva G, Subramanian S, Qadir S, et al. Imaging of the aortic valve using fluorodeoxyglucose positron emission tomography increased valvular fluorodeoxyglucose uptake in aortic stenosis. J Am Coll Cardiol. 2011;57:2507-2515.

10. Dweck MR, Jones C, Joshi NV, et al. Assessment of valvular calcification and inflammation by positron emission tomography in patients with aortic stenosis. Circulation. 2012;125:76-86.

11. Bossé Y, Miqdad A, Fournier D, Pepin A, Pibarot P, Mathieu P. Refining molecular pathways leading to calcific aortic valve stenosis by studying gene expression profile of normal and calcified stenotic human aortic valves. Circ Cardiovasc Genet. 2009;2:489-498.

12. Su H, Spinale FG, Dobrucki LW, et al. Noninvasive targeted imaging of matrix metalloproteinase activation in a murine model of postinfarction remodeling. Circulation. 2005;112:3157-3167.

13. Zhang J, Nie L, Razavian M, et al. Molecular imaging of activated matrix metalloproteinases in vascular remodeling. Circulation. 2008;118:1953-1960.

14. Razavian M, Zhang J, Nie L, et al. Molecular imaging of matrix metalloproteinase activation to predict murine aneurysm expansion in vivo. $\mathrm{J} \mathrm{Nucl} \mathrm{Med}$. 2010;51:1107-1115.

15. Razavian M, Nie L, Challa A, et al. Lipid lowering and imaging protease activation in atherosclerosis. $J$ Nucl Cardiol. 2014;21:319-328.

16. Miller JD, Weiss RM, Heistad DD. Calcific aortic valve stenosis: methods, models, and mechanisms. Circ Res. 2011;108:1392-1412.

17. Akat K, Borggrefe M, Kaden JJ. Aortic valve calcification: basic science to clinical practice. Heart. 2009;95:616-623.

18. Chen JH, Simmons CA. Cell-matrix interactions in the pathobiology of calcific aortic valve disease: critical roles for matricellular, matricrine, and matrix mechanics cues. Circ Res. 2011;108:1510-1524.

19. Helske S, Kupari M, Lindstedt KA, Kovanen PT. Aortic valve stenosis: an active atheroinflammatory process. Curr Opin Lipidol. 2007;18:483-491.

20. Hadler-Olsen E, Fadnes B, Sylte I, Uhlin-Hansen L, Winberg JO. Regulation of matrix metalloproteinase activity in health and disease. FEBS J. 2011;278:28-45.

21. Koppisetti RK, Fulcher YG, Jurkevich A, et al. Ambidextrous binding of cell and membrane bilayers by soluble matrix metalloproteinase-12. Nat Commun. 2014;5:5552.

22. Edep ME, Shirani J, Wolf P, Brown DL. Matrix metalloproteinase expression in nonrheumatic aortic stenosis. Cardiovasc Pathol. 2000;9:281-286.

23. Satta J, Oiva J, Salo T, et al. Evidence for an altered balance between matrix metalloproteinase- 9 and its inhibitors in calcific aortic stenosis. Ann Thorac Surg. 2003;76:681-688.

24. Fondard O, Detaint D, Iung B, et al. Extracellular matrix remodelling in human aortic valve disease: the role of matrix metalloproteinases and their tissue inhibitors. Eur Heart J. 2005;26:1333-1341.

25. Galis ZS, Khatri JJ. Matrix metalloproteinases in vascular remodeling and atherogenesis: the good, the bad, and the ugly. Circ Res. 2002;90:251-262.

26. Hakuno D, Kimura N, Yoshioka M, et al. Periostin advances atherosclerotic and rheumatic cardiac valve degeneration by inducing angiogenesis and MMP production in humans and rodents. J Clin Invest. 2010;120:2292-2306.

27. Clark-Greuel JN, Connolly JM, Sorichillo E, et al. Transforming growth factorbeta1 mechanisms in aortic valve calcification: increased alkaline phosphatase and related events. Ann Thorac Surg. 2007;83:946-953.

28. Kaden JJ, Dempfle CE, Kilic R, et al. Influence of receptor activator of nuclear factor kappa B on human aortic valve myofibroblasts. Exp Mol Pathol. 2005;78:36-40.

29. Balachandran K, Sucosky P, Jo H, Yoganathan AP. Elevated cyclic stretch alters matrix remodeling in aortic valve cusps: implications for degenerative aortic valve disease. Am J Physiol Heart Circ Physiol. 2009;296:H756-H764. 